\title{
Chaotic-Integrable Transition in the Sachdev-Ye-Kitaev Model
}

\author{
Antonio M. García-García, ${ }^{1, \dagger}$ Bruno Loureiro, ${ }^{2, \ddagger}$ Aurelio Romero-Bermúdez, ${ }^{3, *}$ and Masaki Tezuka ${ }^{4, \S}$ \\ ${ }^{1}$ Shanghai Center for Complex Physics, Department of Physics and Astronomy, Shanghai Jiao Tong University, \\ Shanghai 200240, China \\ ${ }^{2}$ TCM Group, Cavendish Laboratory, University of Cambridge, JJ Thomson Avenue, Cambridge CB3 OHE, United Kingdom \\ ${ }^{3}$ Instituut-Lorentz for Theoretical Physics $\triangle I T P$, Leiden University, Niels Bohrweg 2, Leiden 2333CA. The Netherlands \\ ${ }^{4}$ Department of Physics, Kyoto University, Kyoto 606-8502, Japan
}

(Received 16 September 2017; revised manuscript received 5 May 2018; published 15 June 2018)

\begin{abstract}
Quantum chaos is one of the distinctive features of the Sachdev-Ye-Kitaev (SYK) model, $N$ Majorana fermions in $0+1$ dimensions with infinite-range two-body interactions, which is attracting a lot of interest as a toy model for holography. Here we show analytically and numerically that a generalized SYK model with an additional one-body infinite-range random interaction, which is a relevant perturbation in the infrared, is still quantum chaotic and retains most of its holographic features for a fixed value of the perturbation and sufficiently high temperature. However, a chaotic-integrable transition, characterized by the vanishing of the Lyapunov exponent and spectral correlations given by Poisson statistics, occurs at a temperature that depends on the strength of the perturbation. We speculate about the gravity dual of this transition.
\end{abstract}

DOI: 10.1103/PhysRevLett.120.241603

Motivated by its potential applications in high-energy and condensed matter physics, and also because of its simplicity, research on fermionic models with infiniterange random interactions [1-9], now generally called Sachdev-Ye-Kitaev (SYK) models [10-13], has flourished in recent times [11,14-34]. Interesting research lines currently being investigated include not only applications in holography [10-13] but also in random matrix theory [25-27,30,32,34], possible experimental realizations $[19,35,36]$, and extensions involving nonrandom couplings $[24,28]$, higher spatial dimensions $[18,21,31,37,38]$, and several flavors [39]. A natural question to ask [18,21, 24,31,37-39] is to what extent holographic properties are present in generalized SYK models. For instance, similar features are observed for nonrandom couplings [24] and in higher-dimensional realizations of the SYK $[37,38]$ model. However, in some cases, the addition of more fermionic species can induce a transition to a Fermi liquid phase [31] or a metal-insulator transition [18,21], which, at least superficially, spoils a holographic interpretation. Here we study the stability of chaos and holographic features of a generalized SYK model consisting of $N$ fermions in $0+1$ dimension with infinite-range two-body random interaction perturbed by a one-body random term

Published by the American Physical Society under the terms of the Creative Commons Attribution 4.0 International license. Further distribution of this work must maintain attribution to the author(s) and the published article's title, journal citation, and DOI. Funded by SCOAP.

$$
H=\frac{1}{4 !} \sum_{i, j, k, l=1}^{N} J_{i j k l} \chi_{i} \chi_{j} \chi_{k} \chi_{l}+\frac{i}{2 !} \sum_{i, j=1}^{N} \kappa_{i j} \chi_{i} \chi_{j},
$$

where $\chi_{i}$ are Majorana fermions so $\left\{\chi_{i}, \chi_{j}\right\}=\delta_{i j}$. The couplings $J_{i j k l}$ and $\kappa_{i j}$ are Gaussian-distributed random variables with zero average and standard deviation $\left(\sqrt{6} J / N^{3 / 2}\right),(\kappa / \sqrt{N})$, respectively $[10,12]$. We study the model $[12,13]$ by introducing replica fields, averaging over disorder and decoupling the replica fields by two Hubbard-Stratonovich transformations that allow the integration of the original fermionic variables. The resulting partition function is expressed in terms of the bilocal fields $G\left(\tau_{1}, \tau_{2}\right)$ and $\Sigma\left(\tau_{1}, \tau_{2}\right): Z=\int[D G][D \Sigma] e^{-N S_{\text {eff }}}$, where

$$
\begin{aligned}
S_{\text {eff }}= & -\frac{1}{2} \operatorname{Tr} \log \left(\partial_{\tau}-\Sigma\right)+\frac{1}{2} \int d \tau d \tau^{\prime}\left[G\left(\tau, \tau^{\prime}\right) \Sigma\left(\tau, \tau^{\prime}\right)\right. \\
& \left.-\frac{J^{2}}{4} G\left(\tau, \tau^{\prime}\right)^{4}-\frac{\kappa^{2}}{2} G\left(\tau, \tau^{\prime}\right)^{2}\right] .
\end{aligned}
$$

The saddle point equations in imaginary time, which become exact in the large- $N$ limit of interest, are

$G_{n}^{-1}=-i \omega_{n}-\Sigma_{n}, \quad \Sigma(\tau)=-J^{2} G(\tau)^{2} G(-\tau)+\kappa^{2} G(\tau)$,

where $\omega_{n}=(2 \pi / \beta)(n+1 / 2), G_{n} \equiv G\left(i \omega_{n}\right)$, and $\Sigma_{n} \equiv$ $\Sigma\left(i \omega_{n}\right)$. In the long time, strong coupling limit, where conformal symmetry holds, the solution of the SchwingerDyson (SD) equations is dominated by the one-body term 
and therefore $[10,12]$ the zero-temperature entropy always vanishes. The low-temperature limit of the specific heat is directly related to the leading correction to the conformal Green's function. A simple power-counting argument in the SD equations suggests that it has contributions from both terms. Therefore, we expect the specific heat still to be linear with a slope $c$ that may depend on $\kappa$. We confirm these results by exact diagonalization of Eq. (1) with $J=1$. For a given set of parameters, we have obtained at least $10^{6}$ eigenvalues. We have computed, following $[26,30]$, the entropy at zero-temperature $s_{0}$ and the specific heat by using standard thermodynamic relations and a finite-size scaling analysis. As was expected, we have found a vanishing $s_{0}$ for any $\kappa$ and a linear specific heat with $c \propto N f(\kappa)$ with $f \sim 0.5 / \kappa$ for large $\kappa$ and a steady increase for smaller $\kappa$. We note that all these features, including $s_{0}=0$ [40], are consistent with the existence of a gravity dual. We have confirmed these results by an explicit evaluation of the free energy from Eqs. (2) and (3). See Supplemental Material, Secs. A and B [41], for more details. Next we employ level statistics [42] to investigate the effect of the one-body perturbation in the quantum chaotic features of the model.

Level statistics.-For that purpose, we compute, from the exact diagonalization of Eq. (1), the level spacing distribution $P(s)$, the probability to find two consecutive eigenvalues $E_{i}, E_{i+1}$ at a distance $s=\left(E_{i+1}-E_{i}\right) / \Delta$, that probes the system dynamics for times of the order of the Heisenberg time $\sim \hbar / \Delta$ with $\Delta$ the mean level spacing. For an insulator, or a generic-integrable system, it is given by Poisson statistics, $P_{P}(s)=e^{-s}$ [42], while for a quantum chaotic system, it is given by WD statistics [43], which is well approximated by the Wigner surmise, $P_{W}(s) \approx\left(32 / \pi^{2}\right) s^{2} \exp \left(-4 s^{2} / \pi\right)$, for systems with broken time reversal invariance [42]. For a meaningful comparison with these predictions, unfolding the spectrum [42] is necessary so that $\Delta=1$ by a local fitting of the numerical spectral density by a smooth function, which is subsequently employed to rescale the spectrum.

Results for $P(s)$ as a function of $\kappa$, depicted in Fig. 1, show a gradual crossover from Poisson to WD statistics as $\kappa$ decreases, which is also observed in the tail of the distribution (see inset). Unlike the standard SYK model $[25,26]$, the one-body term breaks time reversal invariance for all $N$. As was mentioned previously, for $J=0$, the Hamiltonian is effectively noninteracting, which suggests that Poisson statistics applies in the $N \rightarrow \infty$ limit. In order to determine whether Poisson statistics is robust for $J \gg \kappa$, and therefore a transition occurs at a finite $\kappa$, we carry out a finite-size scaling analysis employing as scaling variable the adjacent gap ratio [44-46],

$$
r_{i}=\frac{\min \left(\delta_{i}, \delta_{i+1}\right)}{\max \left(\delta_{i}, \delta_{i+1}\right)}
$$
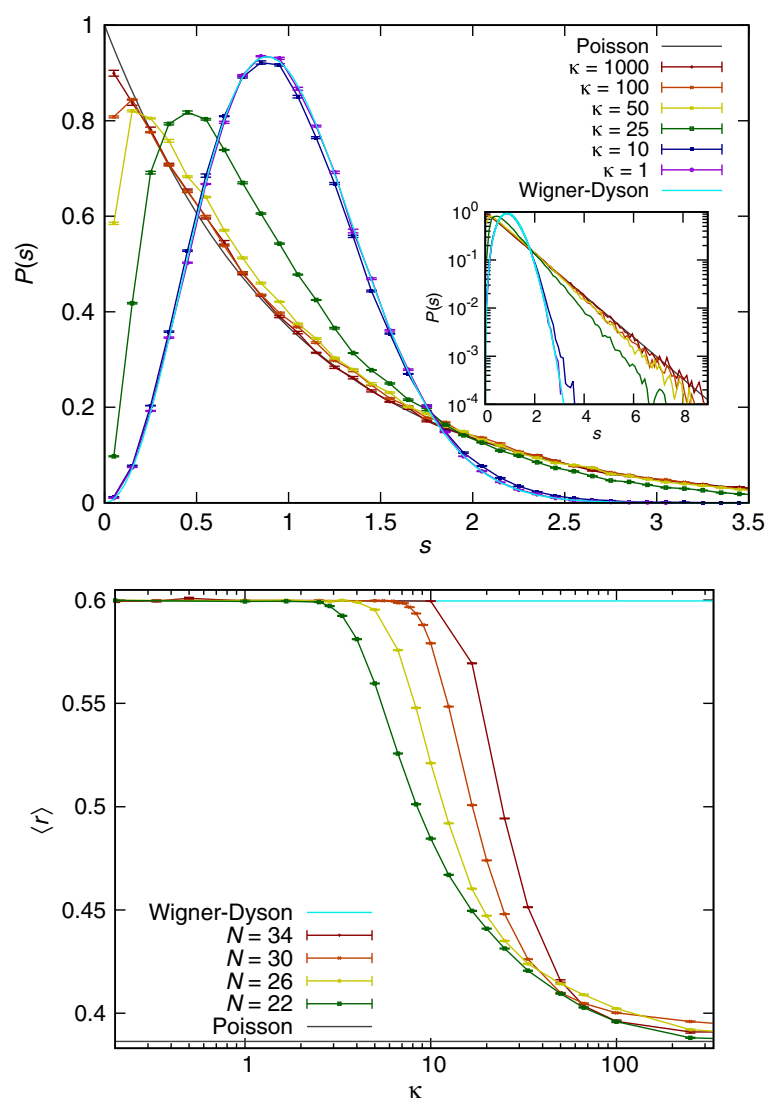

FIG. 1. (Upper) $P(s)$ for $N=34$ and different $\kappa$ 's with $\kappa$ in units of $J=1$. We clearly observe a crossover from WignerDyson (WD) to Poisson statistics as $\kappa$ increases. (Lower) Finitesize scaling analysis of the averaged adjacent gap ratio $\langle r\rangle$ [Eq. (4)] as a function of $\kappa$ for different $N$ 's. For sufficiently large $N$, we observe a crossing at $\kappa_{c} \approx 66$, which suggests the existence of a chaotic-integrable transition. Results for larger $N$ would be necessary to confirm it. See the main text for an explanation of the absence of crossing for small $N$. Both $P(s)$ and $\langle r\rangle$ were computed by using ensemble and spectral average in a window comprising $10 \%$ of the eigenvalues around the center of the spectrum. Results are robust to changes in the percentage of eigenvalues provided that the spectrum edges are avoided.

for an ordered spectrum $E_{i-1}<E_{i}<E_{i+1}$, where $\delta_{i}=$ $E_{i}-E_{i-1}$. The average adjacent gap ratio for a Poisson distribution is $\langle r\rangle_{P}=2 \ln (2)-1 \approx 0.386$, while for WD statistics, it is $\approx 0.599$ [47]. In Fig. $1,\langle r\rangle$ is depicted as a function of $\kappa$ for different $N$ 's. Only $10 \%$ of the total number of eigenvalues, located around the center of the spectrum, are employed in the calculation. We stick to values of $N$ for which time reversal symmetry is broken even in the absence of the one-body term in Eq. (1). As was expected, except for $\kappa \gg 10,\langle r\rangle$ is very close to the WD result for any $N$. Only for $\kappa \geq 25$, a crossover to Poisson statistics is observed. For $N \leq 22$, we did not observe a crossing point in the plot, and moreover, $\langle r\rangle$ gradually approaches the WD prediction, which suggests that the system is quantum chaotic for any $\kappa$. However, for $N \geq 30$, 
we observe a crossing at $\kappa=\kappa_{c} \approx 60$. This is an indication of a transition from chaos to integrability, though it would be necessary to explore larger $N$ 's to confirm it. A possible explanation for the different behavior for small $N$ is that the lowest eigenvalues (the most infrared part of the spectrum) are strongly correlated. The reason is that the one-particle sector for $\kappa \rightarrow \infty$, which controls the lowest energy properties, is known [30] to be described by a skeworthogonal random matrix. The number of eigenvalues related to one-particle states decreases exponentially with $N$, which would explain why its contribution is only relevant for sufficiently small $N$.

We note the existence of the gravity dual is related to the properties of the model in the low-temperature, strong coupling limit described by the tail, not the bulk of the spectrum studied above. Moreover, we are also interested in the nature of level statistics for shorter timescales, where random matrix theory predicts level rigidity [42]. In order to investigate these issues, we compute the connected spectral form factor of the unfolded spectrum

$$
g_{c}(t) \equiv\left\langle\frac{Z(t, \beta) Z^{*}(t, \beta)}{Z(0, \beta)^{2}}\right\rangle-\left|\left\langle\frac{Z(t, \beta)}{Z(0, \beta}\right\rangle\right|^{2},
$$

where $Z(t, \beta)=\operatorname{Tr} e^{-\beta H-i H t}$ and $\beta>0$. For quantum chaotic systems, and also for the unperturbed SYK model [26,30], we expect a correlation hole [30,48-51] for intermediate times followed by a ramp, related to the level rigidity observed in quantum chaotic systems [42]. We note that, by increasing $\beta$, we probe the tail of the spectrum. Results, depicted in Fig. 2, show that for $\kappa=1$ the ramp is still observed for sufficiently small $\beta$. In order to clarify the situation for larger $\beta$, which probes the spectral correlations of the smallest eigenvalues, we again carry out a finite-size scaling analysis of the averaged adjacent gap ratio $\langle r\rangle$ [Eq. (4)], but the average is weighted by $\beta$ (see caption of Fig. 2), so that the low energy part of the spectrum is singled out.

A crossing seems to be observed (see lower plot of Fig. 2) at $\kappa=\kappa_{c} \approx 25$. This is a signature of a chaos-integrable transition in the tail of the spectrum. However, results are not conclusive because the size dependence is weak for large $\kappa$. We only note that, in qualitative agreement with the results of next section, $\kappa_{c}$ for $\beta=0.2$ is smaller than in Fig. 1, where effectively $\beta \approx 0$. It is worth mentioning that similar chaoticintegrable transitions in level statistics have been previously studied in the context of nuclear physics [52] and quantum chaos [7,53-55] in somehow related models, such as complex fermions with infinite-range interactions and a random diagonal one-body term or interacting systems with short-range interactions [56].

In summary, the finite-size scaling analysis is not fully conclusive to detect the chaotic-integrable transition. In order to confirm it, we investigate next out-of-time-order four-point correlation functions where quantum chaotic
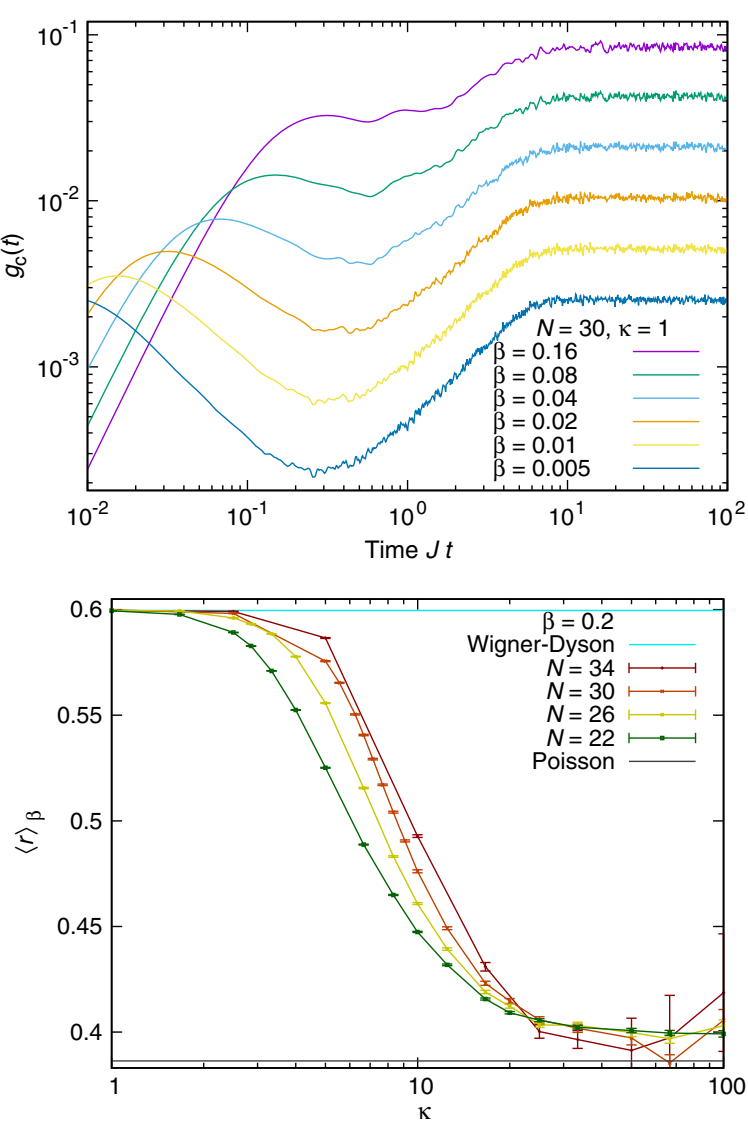

FIG. 2. (Upper) Connected spectral form factor $g_{c}(t)$ for the unfolded spectrum from Eq. (5) for $N=30, J=\kappa=1$, and different $\beta$ 's. For small $\beta$, we observe the correlation hole $[48,49]$ followed by a ramp typical of quantum chaotic systems. As $\beta$ increases, which probes the tail of the spectrum, results are not conclusive. (Lower) A finite-size scaling analysis, also with $J=1$, of the average adjacent gap ratio $\langle r\rangle_{\beta}$ [Eq. (4)], where, unlike the previous figure, the average is weighted by the function $\exp \left[-\beta\left(E_{i}+2 E_{i+1}+E_{i+2}\right) / 4\right]$, but the spectrum is not unfolded. Here we have excluded the ten smallest eigenvalues from the analysis because $\langle r\rangle$ for such eigenvalues at the spectral tail is anomalous high even in the large- $\kappa$ limit. For details, see the Supplemental Material [41]. For $\beta=0.2$, which probes the low energy part of the spectrum, we observe a crossing at $\kappa_{c} \approx 25$ that seems to indicate a chaotic-integrable transition. However, the size dependence is too weak to confirm the existence of the transition.

features are characterized by a finite Lyapunov exponent [57-59].

Out-of-time-order four-point correlation function.-In the semiclassical limit, the time evolution of certain out-oftime-order correlation functions experiences a period of exponential growth $[59,60]$ around the Ehrenfest time $t_{*} \sim \lambda_{L}^{-1} \log \left(\hbar / S_{0}\right)$, where $S_{0}$ is a typical action of the system and $\lambda_{L}$ is the classical Lyapunov exponent. By contrast, for nonchaotic systems, the growth of $t_{*}$ with $\hbar$ is only power law [61]. The application of these ideas in highenergy physics, where $\hbar$ is traded by a parameter $\sim 1 / N$ that 
controls small quantum gravity corrections, has led to the proposal that black holes are quantum chaotic [57] with a Lyapunov exponent that saturates a recently proposed universal bound $\lambda_{L} \leq 2 \pi k_{B} T / \hbar$ [58]. We now study whether these chaotic features are present in Eq. (1). We compute $\lambda_{L}$ from the following $[12,58,62]$ out-of-timeorder correlator

$$
\begin{aligned}
F\left(t_{1}, t_{2}\right) \equiv & \frac{1}{N^{2}} \sum_{i, j}^{N} \operatorname{Tr}\left[\rho(\beta)^{\frac{1}{4}} \chi_{i}\left(t_{1}\right) \rho(\beta)^{\frac{1}{4}} \chi_{j}(0)\right. \\
& \left.\times \rho(\beta)^{\frac{1}{4}} \chi_{i}\left(t_{2}\right) \rho(\beta)^{\frac{1}{4}} \chi_{j}(0)\right] \\
\simeq & G_{R}\left(t_{1}\right) G_{R}\left(t_{2}\right)+\frac{1}{N} \mathcal{F}\left(t_{1}, t_{2}\right)+\mathcal{O}\left(\frac{1}{N^{2}}\right),
\end{aligned}
$$

where $\rho^{1 / 4}(\beta)=\left[\left(e^{-\beta H} / Z\right)^{1 / 4}\right]$ is inserted [58] along the thermal cycle to regularize the otherwise divergent operator. It is possible to show that $\mathcal{F}\left(t_{1}, t_{2}\right)$ satisfies

$$
\begin{array}{r}
\mathcal{F}\left(t_{1}, t_{2}\right)=\int d t_{3} d t_{4} K_{R}\left(t_{1}, t_{2}, t_{3}, t_{4}\right) \mathcal{F}\left(t_{3}, t_{4}\right), \\
K_{R}\left(t_{1}, t_{2}, t_{3}, t_{4}\right)=G_{R}\left(t_{1}\right) G_{R}\left(t_{2}\right)\left[3 J^{2} G_{l r}^{2}\left(t_{3}-t_{4}\right)+\kappa^{2}\right],
\end{array}
$$

where $G_{R}(\omega)=G\left(i \omega_{n} \rightarrow \omega+i 0^{+}\right)$is the retarded Green's function in real frequency and $G_{l r}(t)$ is the Wightman function obtained from $G_{l r}(\omega)=\left[2 i e^{-\beta \omega / 2} /\left(1+e^{-\beta \omega}\right) \times\right.$ ]$\Im\left[G_{R}(\omega)\right]$. In order to compute these two-point functions, we follow the strategy employed in Refs. [12,31]. We analytically continue $i \omega_{n} \rightarrow \omega+i 0^{+}$the saddle point equations (3) and solve them using the spectral representation of the retarded Green's function. Substituting the ansatz $\mathcal{F}\left(t_{1}, t_{2}\right)=e^{\lambda_{L}\left(t_{1}+t_{2}\right) / 2} f\left(t_{12}\right)$, where $t_{12}=t_{1}-t_{2}$, into Eq. (7) and expressing it in the frequency domain, we obtain the following eigenvalue equation for $f(\omega)$,

$$
\begin{aligned}
f\left(\omega^{\prime}\right)= & \left|G^{R}\left(\omega^{\prime}+i \frac{\lambda_{L}}{2}\right)\right|^{2} \\
& \times\left[\kappa^{2} f\left(\omega^{\prime}\right)+3 J^{2} \int \frac{d \omega}{2 \pi} g_{l r}\left(\omega^{\prime}-\omega\right) f(\omega)\right],
\end{aligned}
$$

where $\omega^{\prime}=\omega_{1}-i \lambda_{L} / 2$ and $g_{l r}(\omega)=\int d t e^{i \omega t} G_{l r}(t)^{2}$. Finally, we compute $\lambda_{L}$ by imposing the existence of a nondegenerate eigenvalue equal to one so that Eq. (9) is satisfied.

Results, depicted in Fig. 3, show the system displays chaotic behavior, namely, the Lyapunov exponent $\lambda_{L}$ is finite, for all studied values of $\kappa$ and sufficiently high temperature. However, even in the strong coupling limit, $\lambda_{L}$ never approaches the bound $\lambda_{L}=2 \pi k_{B} T / \hbar$. Indeed, for a given temperature, $\lambda_{L}$ decreases as $\kappa$ increases and eventually vanishes for sufficiently strong $\kappa$ or, for a fixed $\kappa$, for sufficiently low temperature. Therefore, quantum chaos is

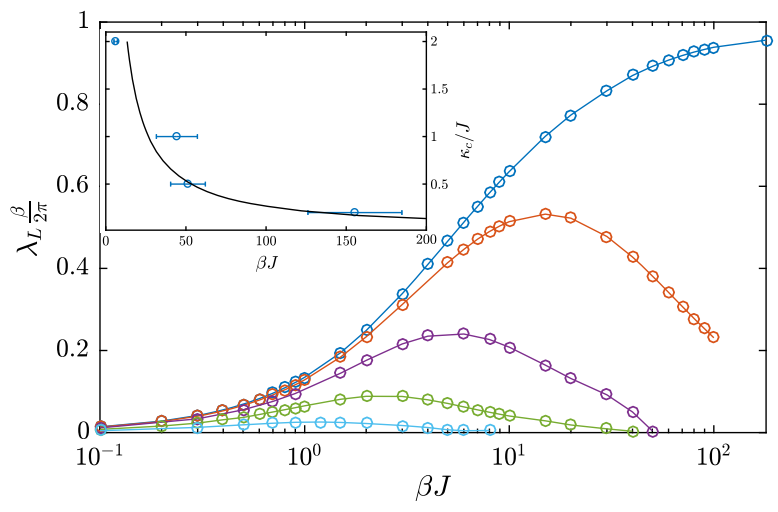

FIG. 3. Lyapunov exponent $\lambda_{L}$ for the model Eq. (1) with $J=1$ as a function of the inverse temperature $\beta=1 / T$ and $\kappa$. From top to bottom, $\kappa=0,0.2,0.5,1,2$. A finite $\lambda_{L}$, which is a signature of quantum chaos, is observed for a fixed $\kappa$ and not too low temperature. For sufficiently low temperatures, and $\kappa>0$, we identify a $T^{*}(\kappa)$ such that $\lambda_{L}=0$ for any $T<T^{*}(\kappa)$, which signals a chaotic-integrable transition. (Inset) Critical value of $\kappa=\kappa_{c}$ at which the transition takes place as a function of $\beta$. Dots result from fitting the numerical data of the main plot near the transition. The solid line is the analytical expression from Eq. (15) valid in the large- $q$ limit. Agreement with the numerical data is reasonable for $\kappa / J \ll 1$.

robust to the introduction of a relevant one-body perturbation but only if it is weak enough and the temperature is high enough. We now confirm these results analytically by studying the following model with $q$ /2-body interactions,

$H=i^{\frac{q}{2}} \sum_{1 \leq i_{1}<i_{2}<\ldots<i_{q} \leq N} J_{i_{1}, i_{2}, \ldots, i_{q}} \chi_{i_{1}} \chi_{i_{2}} \ldots \chi_{i_{q}}+i \sum_{1 \leq i<j \leq N} \kappa_{i j} \chi_{i} \chi_{j}$,

where $\kappa_{i j}$ and $J_{i_{1} i_{2}, \ldots, i_{q}}$ are again Gaussian-distributed random variables with zero average and $q$-dependent variances $\left(\kappa^{2} / q N\right),\left(2^{q-1} / q\right)\left[(q-1) ! J^{2} / N^{q-1}\right]$, and $q \gg 1$, respectively. As before, we fix $J$ and use $\kappa$ and $\beta$ as the only parameters. The key insight is that the retarded kernel in this model, given by

$$
\begin{aligned}
& K_{R}\left(t_{1}, t_{2}, t_{3}, t_{4}\right) \\
& \quad=\frac{G_{R}\left(t_{1}\right) G_{R}\left(t_{2}\right)}{q}\left[(q-1) J^{2} G_{l r}\left(t_{3}-t_{4}\right)^{q-2}+\kappa^{2}\right],
\end{aligned}
$$

simplifies considerably in the limit of $q \gg 1$, allowing for an analytical solution of the eigenvalue problem in Eq. (7). First, we proceed in the same way as for the model (1) to find an effective action and the associated saddle point equations analogous to Eqs. (2) and (3). In the limit $q \gg 1$, the saddle point equations can be consistently expanded in terms of

$$
G(\tau) \underset{q \gg 1}{=} \frac{1}{2} \operatorname{sgn}(\tau)\left[1+q^{-1} g(\tau)+O\left(q^{-2}\right)\right],
$$


yielding a nonlinear boundary value problem for $g$,

$$
\partial_{\theta}^{2} g=2(\beta J)^{2} e^{g(\theta)}+(\beta \kappa)^{2},
$$

with $\theta=\tau / \beta \in(0,1)$. The retarded kernel Eq. (11) is thus obtained by analytical continuation of the resulting $G(\tau)$ and, as mentioned before, for $q \gg 1$, it is given by a simpler expression,

$K_{R}\left(t_{1}, t_{2}, t_{3}, t_{4}\right)=\theta\left(t_{13}\right) \theta\left(t_{24}\right)\left(2 J^{2} e^{g\left(\tau=i t_{34}+\beta / 2\right)}+q^{-1} \kappa^{2}\right)$,

where $g(\tau)$ is the solution of Eq. (13) and is given explicitly as a power series in $\kappa / J \ll 1$ in the Supplemental Material, Sec. C [41]. We again use the ansatz $\mathcal{F}\left(t_{1}, t_{2}\right)=$ $e^{\lambda_{L}\left(t_{1}+t_{2}\right) / 2} f\left(t_{12}\right)$ in order to rewrite the eigenvalue problem in Eq. (7) as a Schrödinger equation for $f\left(t_{12}\right)$. The eigenstates of the resulting equation are found perturbatively in $\kappa / J \ll 1$, giving a correction $O\left(\kappa^{2}\right)$ to the Lyapunov exponent. This correction is given in terms of an integral that, for low temperature $\beta J \gg 1$, is approximated by

$\left.\frac{\beta \lambda_{L}}{2 \pi}\right|_{\substack{q \gg 1 \\ \kappa \ll J}}=1-\frac{(\beta \kappa)^{2}}{\pi^{2}}\left[\frac{1}{72}+\frac{19-18 \log \pi}{36 \beta J}+O\left(\frac{1}{(\beta J)^{2}}\right)\right]$.

The transition occurs when $\lambda_{L}=0$, which leads to a $\beta$ dependent critical $\kappa=\kappa_{c}$. For instance, $\kappa_{c}(\beta=133) \sim 0.2 J$ and $\kappa_{c}(\beta=53) \sim 0.5 \mathrm{~J}$, which is in good agreement (see Fig. 3) with numerical results for $q=4$. We refer to Sec. C of the Supplemental Material [41] for additional details.

Finally, we note these types of transitions are generic [56], so it would be interesting to identify their gravity dual. We speculate with the possibility that the gravity dual of the transition studied in this Letter is a Hawking-Page transition, where the black hole and thermal gas phases correspond to the chaotic and integrable phase, respectively. In conclusion, we have found that the SYK model perturbed by a random one-body term is still chaotic in the limit of sufficiently high temperature or weak perturbation. However, for a given strength of the perturbation, the system undergoes a chaotic-to-integrable transition for sufficiently low temperatures, which may have a gravity dual interpretation.

We thank D. Anninos, S. Banerjee, and $P$. Sabella-Garnier for illuminating discussions. Part of the computation in this Letter has been done using the facilities of the Supercomputer Center, the Institute for Solid State Physics, the University of Tokyo. A. M. G. acknowledges partial financial support from a QuantEmX grant from Institute for Complex Adaptive Matter (ICAM) and the Gordon and Betty Moore Foundation through Grant No. GBMF5305. The work of M. T. was partially supported by Grants-in-Aid No. JP26870284 and No. JP17K17822 from JSPS of Japan. A. R. B. is funded through a research program of the Foundation for Fundamental Research on Matter (FOM), which is part of the Netherlands Organisation for Scientific Research (NWO). B. L. is supported by a CAPES/Cambridge Overseas Trust (COT) Grant No. 11469/13-17.

Note added.-Recently, we became aware of three papers [63-65] that study a somehow similar generalized SYK model, though the focus of these papers is rather different. Reference [65] studies quantum quenches, while the other two investigate a two-fermion species generalization of the model in which a transition occurs by tuning the number of fermions.

*romero@lorentz.leidenuniv.nl

†amgg@sjtu.edu.cn

tbl360@cam.ac.uk

\$tezuka@scphys.kyoto-u.ac.jp

[1] O. Bohigas and J. Flores, Phys. Lett. B 34, 261 (1971).

[2] O. Bohigas and J. Flores, Phys. Lett. B 35, 383 (1971).

[3] J. French and S. Wong, Phys. Lett. B 33, 449 (1970).

[4] J. French and S. Wong, Phys. Lett. B 35, 5 (1971).

[5] K. Mon and J. French, Ann. Phys. (N.Y.) 95, 90 (1975).

[6] L. Benet and H. A. Weidenmüller, J. Phys. A 36, 3569 (2003).

[7] V. K. B. Kota, Embedded Random Matrix Ensembles in Quantum Physics (Springer, New York, 2014), Vol. 884.

[8] S. Sachdev and J. Ye, Phys. Rev. Lett. 70, 3339 (1993).

[9] S. Sachdev, Phys. Rev. Lett. 105, 151602 (2010).

[10] A. Kitaev, A Simple Model of Quantum Holography, KITP Program: Entanglement in Strongly-Correlated Quantum Matter, Santa Barbara, 2015, http://online.kitp.ucsb.edu/ online/entangled15/.

[11] K. Jensen, Phys. Rev. Lett. 117, 111601 (2016).

[12] J. Maldacena and D. Stanford, Phys. Rev. D 94, 106002 (2016).

[13] S. Sachdev, Phys. Rev. X 5, 041025 (2015).

[14] A. Almheiri and J. Polchinski, J. High Energy Phys. 11 (2015) 014.

[15] J. Maldacena, D. Stanford, and Z. Yang, Prog. Theor. Exp. Phys. 2016, 12C104 (2016).

[16] J. Engelsöy, T. G. Mertens, and H. Verlinde, J. High Energy Phys. 07 (2016) 139.

[17] D. Bagrets, A. Altland, and A. Kamenev, Nucl. Phys. B911, 191 (2016).

[18] C.-M. Jian, Z. Bi, and C. Xu, Phys. Rev. B 96, 115122 (2017).

[19] I. Danshita, M. Hanada, and M. Tezuka, Prog. Theor. Exp. Phys. 2017, 083I01 (2017).

[20] A. Jevicki, K. Suzuki, and J. Yoon, J. High Energy Phys. 07 (2016) 007.

[21] S.-K. Jian and H. Yao, Phys. Rev. Lett. 119, 206602 (2017).

[22] J. M. Magán, Phys. Rev. Lett. 116, 030401 (2016).

[23] J. M. Magán, J. High Energy Phys. 08 (2016) 81.

[24] E. Witten, arXiv:1610.09758. 
[25] Y.-Z. You, A. W. W. Ludwig, and C. Xu, Phys. Rev. B 95, 115150 (2017).

[26] A. M. García-García and J. J. M. Verbaarschot, Phys. Rev. D 94, 126010 (2016).

[27] A. M. García-García and J. J. M. Verbaarschot, Phys. Rev. D 96, 066012 (2017).

[28] I. R. Klebanov and G. Tarnopolsky, Phys. Rev. D 95, 046004 (2017).

[29] D. Bagrets, A. Altland, and A. Kamenev, Nucl. Phys. B921, 727 (2017).

[30] J. S. Cotler, G. Gur-Ari, M. Hanada, J. Polchinski, P. Saad, S. H. Shenker, D. Stanford, A. Streicher, and M. Tezuka, J. High Energy Phys. 05 (2017) 118.

[31] S. Banerjee and E. Altman, Phys. Rev. B 95, 134302 (2017).

[32] T. Kanazawa and T. Wettig, J. High Energy Phys. 09 (2017) 50.

[33] C. Krishnan, S. Sanyal, and P. N. B. Subramanian, J. High Energy Phys. 03 (2017) 56.

[34] C. Krishnan, K. V. P. Kumar, and S. Sanyal, J. High Energy Phys. 06 (2017) 036.

[35] L. García-Álvarez, I. L. Egusquiza, L. Lamata, A. del Campo, J. Sonner, and E. Solano, Phys. Rev. Lett. 119, 040501 (2017).

[36] D. I. Pikulin and M. Franz, Phys. Rev. X 7, 031006 (2017).

[37] Y. Gu, X.-L. Qi, and D. Stanford, J. High Energy Phys. 05 (2017) 125.

[38] R. A. Davison, W. Fu, A. Georges, Y. Gu, K. Jensen, and S. Sachdev, Phys. Rev. B 95, 155131 (2017).

[39] D. J. Gross and V. Rosenhaus, J. High Energy Phys. 02 (2017) 93.

[40] B. Goutéraux and E. Kiritsis, J. High Energy Phys. 12 (2011) 36.

[41] See Supplemental Material at http://link.aps.org/ supplemental/10.1103/PhysRevLett.120.241603 for (1) thermodynamic properties numerically for finite and large- $N$ and analytically using perturbation theory for large$N$, (2) the Lyapunov exponent perturbatively and (3) anomalous values of $\langle r\rangle$ in the infrared limit of the spectrum.

[42] T. Guhr, A. Mueller-Groeling, and H. A. Weidenmueller, Phys. Rep. 299, 189 (1998).

[43] M. L. Mehta, Random Matrices (Academic Press, New York, 2004).
[44] D. J. Luitz, N. Laflorencie, and F. Alet, Phys. Rev. B 91, 081103 (2015).

[45] V. Oganesyan and D. A. Huse, Phys. Rev. B 75, 155111 (2007).

[46] C. L. Bertrand and A. M. García-García, Phys. Rev. B 94, 144201 (2016).

[47] Y. Y. Atas, E. Bogomolny, O. Giraud, and G. Roux, Phys. Rev. Lett. 110, 084101 (2013).

[48] Y. Alhassid and R. D. Levine, Phys. Rev. A 46, 4650 (1992).

[49] E. J. Torres-Herrera, A. M. García-García, and L. F. Santos, Phys. Rev. B 97, 060303 (2018).

[50] A. Kudrolli, S. Sridhar, A. Pandey, and R. Ramaswamy, Phys. Rev. E 49, R11 (1994).

[51] H. Alt, H.-D. Gräf, T. Guhr, H. L. Harney, R. Hofferbert, H. Rehfeld, A. Richter, and P. Schardt, Phys. Rev. E 55, 6674 (1997).

[52] S. Åberg, Phys. Rev. Lett. 64, 3119 (1990).

[53] P. Jacquod and D. L. Shepelyansky, Phys. Rev. Lett. 79, 1837 (1997).

[54] R. Berkovits and Y. Avishai, Phys. Rev. Lett. 80, 568 (1998).

[55] V. K. B. Kota, A. Relaño, J. Retamosa, and M. Vyas, J. Stat. Mech. (2011) P10028.

[56] M. Di Stasio and X. Zotos, Phys. Rev. Lett. 74, 2050 (1995).

[57] Y. Sekino and L. Susskind, J. High Energy Phys. 10 (2008) 065.

[58] J. Maldacena, S. H. Shenker, and D. Stanford, J. High Energy Phys. 08 (2016) 106.

[59] A. Larkin and Y. N. Ovchinnikov, Sov. Phys. JETP 28, 1200 (1969).

[60] G. Berman and G. Zaslavsky, Physica (Amsterdam) 91A, 450 (1978).

[61] Y. Lai, E. Ott, and C. Grebogi, Phys. Lett. A 173, 148 (1993).

[62] J. Polchinski and V. Rosenhaus, J. High Energy Phys. 04 (2016) 1.

[63] X.-Y. Song, C.-M. Jian, and L. Balents, Phys. Rev. Lett. 119, 216601 (2017).

[64] X. Chen, R. Fan, Y. Chen, H. Zhai, and P. Zhang, Phys. Rev. Lett. 119, 207603 (2017).

[65] A. Eberlein, V. Kasper, S. Sachdev, and J. Steinberg, Phys. Rev. B 96, 205123 (2017). 